\title{
Acetalization of Cyclohexanone Over Transition Metals Modified Mesoporous Ceria-A Green Approach
}

\author{
Rose Philo K. J' ${ }^{1,}$ Sugunan S. ${ }^{2}$ \\ ',(Department of Chemistry, St.Paul's College, Kalamassery, Kerala, India). \\ 2.(Department of Applied Chemistry, Cochin University of Science and Technology, Kerala, India).
}

\begin{abstract}
The introduction and removal of protecting groups can play an important role in organic and natural product synthesis.The synthesis of dimethyl acetals of carbonyl compound such as cyclohexanone has successfully been carried out by the reaction between cyclohexanone and methanol using different transition metals modified mesoporous ceria catalysts. The strong influence of the textural properties of the catalysts such as acid amount and adsorption properties (surface area and pore volume) determine the catalytic activity. We introduced a mild and selective reagent for the oxidative cleavage of acetals under aqueous and heterogeneous conditions.The stability, simple work-up and high yields of the products are among the advantages of this procedure which make it an attractive supplement to the present reported methods.
\end{abstract}

Keywords: dimethyl acetals, deprotection of acetals, catalytic activity, transition metals modified mesoporous ceria

\section{Introduction}

The title reaction is many times a requirement to protect carbonyl groups specifically during the manipulation of multifunctional organic molecules since dimethyl acetals display higher stability towards strong bases, Grignard reagent, lithium aluminium hydride, strong oxidants and esterification reagents than their parent carbonyl compounds [1]. Besides the interest of acetals as protecting groups of carbonyl compounds during organic synthesis, many of them have found direct applications as fragrances in cosmetics, food and beverage additives, pharmaceuticals, and polymer chemistry, medicinal and drug design chemistry. Although many efficient and reliable reactions for the conversion of carbonyl compounds to their corresponding acetals have been reported, there aresome drawbacks such as long reaction times, tedious work-up and separation of catalyst, toxic metal waste, unwanted side reaction and nonselectivity regarding these methods. Therefore, design and synthesis of a catalytic system that may be stable, easily separable, and reusable has long been pursued. The choice of the catalyst is of prime importance in these environmentally conscious days. Green chemistry demands the replacement of highly corrosive, hazardous and polluting acid catalysts with eco-friendly and renewable solid acid catalysts. The use of heterogeneous solid catalysts in the organic synthesis is interesting and important, since they provide green alternatives to homogeneous catalysts.

\section{Materials and Methods}

2.1 Experimental

Mesoporous ceria was synthesized, according to literature procedure [2], modified with 3 different compositions of $\mathrm{Fe}, \mathrm{Cr}, \mathrm{Ni} \& \mathrm{Cu}$ by wet impregnation method and characterized by various analytical and spectroscopic techniques, viz., low and wide angle powder X-ray diffraction (XRD, Rigaku D MAX III VC), thermo gravimetry-differential thermal analysis (TG/DTA) (Perkin Elmer TG analyzer), inductively coupled plasma - atomic emission spectroscopy (ICP-AES, Labtam Plasma 8440), diffuse reflectance ultraviolet-visible (DRUV-Vis, Shimadzu UV-2101 PC spectrometer), FT-IR spectra (Shimadzu FTIR 8201), SEM analysis (JEOL JSM-840 A (Oxford make) model 16211), TEM (JEOL 30100), TPR- $\mathrm{H}_{2}$ for reducibility of samples and $\mathrm{N}_{2}$ adsorption-desorption studies for surface area and porosity (Micromeritics Tristar 3000 surface area and porosity analyser) were studied. The acidity measurements of the prepared samples were done by Temperature Programmed Desorption (TPD) of ammonia [3] and the values obtained were tested by vapour phase cumene cracking reaction [1].

\subsection{Synthesis and characterization}

In a typical synthesis procedure mesoporous ceria was prepared according to literature procedure [2]. Since the neutral surfactant -inorganic interaction is due to weak hydrogen bonding the removal of most of the surfactant can be done by washing/solvent extraction. The filtered precipitate is dried and then precalcined at $250^{\circ} \mathrm{C}$ for 6 hours to make the calcination effective at lower temperature. The sample is then calcined at $350^{\circ} \mathrm{C}$ for $4 \mathrm{hrs}$. Mesoporous ceria thus obtained was modified by different weight\% (2, 4\&10 wt. \%) of iron, chromium, nickel \& copper by wet impregnation method. 


\subsubsection{Characterization}

Powder X-ray diffractograms of the materials were recorded on a Rigaku D MAX III VC Ni-filtered $\mathrm{Cu} \mathrm{K} \alpha$ radiation, $\lambda=1.5404 \mathrm{~A}^{\circ} 2 \theta$ range $10-80^{\circ}$ at a speed of $1 \% \mathrm{~min}$. The crystalline phases were identified by comparison with standard JCPDS (Joint Committee on Powder Diffraction Standards) data file [4]. Low angle $\mathrm{XRD}$ was also done to confirm the mesoscopic nature of the sample. $\mathrm{N}_{2}$ adsorption-desorption isotherms, pore size distributions as well as the textural properties of the materials were determined at $-196{ }^{\circ} \mathrm{C}$ by a Micromeritics Tristar 3000 surface area and porosity analyser. Prior to the measurements the samples were degassed for $1 \mathrm{~h}$ at $90^{\circ} \mathrm{C}$ followed by $200^{\circ} \mathrm{C}$ overnight. Pore size distribution and average pore size of the samples were obtained from the adsorption branch of the isotherms using the Barrnet- Joyner-Halenda (BJH) method. FTIR spectra of the solid samples were taken in the range of 4000-400 cm-1 on a Shimadzu FTIR 8201 instrument by diffuse reflectance scanning disc technique. Diffuse reflectance UV-Vis spectra were recorded in the range 200-800 nm with a Shimadzu UV-2101 PC spectrometer equipped with a diffuse reflectance attachment, using $\mathrm{BaSO}_{4}$ as the reference. SEM analysis of the samples was done using JEOL JSM-840 A (Oxford make) model16211 scanning electron microscope analyzer with a resolution of $13 \mathrm{eV}$. The HR-TEM of the sample was carried out in ultrahigh resolution analytical electron microscope JEOL 3010. Perkin Elmer TG analyzer instrument was used for carrying out thermo gravimetric studies. About $20 \mathrm{mg}$ of the sample was used at a heating rate of $20^{\circ} \mathrm{C}$ per min in nitrogen atmosphere. The TG data were computer processed to get thermo grams. Elemental composition was confirmed by ICP-AES.

\subsection{Reaction Procedure}

In order to explore the influence of the modified mesoporous ceria catalyst on acetalization reaction, the reaction of cyclohexanone with methanol was chosen as a model reaction. Initially, the reaction was performed under catalyst free condition and no product formation was observed. Then, to obtain optimized reaction conditions, the reaction was performed with cyclohexanone and methanol using different amounts of modified mesoporous ceria catalyst at different temperatures. Cyclohexanone, purchased from Aldrich Chemical Company, USA and commercial-grade methanol (available from S. D. Fine Chemicals, India) were used as received. One-pot acetalization reactions of carbonyl compounds were carried out in a $50 \mathrm{~mL}$ glass batch reactor equipped with a magnetic stirrer, thermometer, water condenser and temperature controller. Samples were withdrawn every 30 minutes and at the end of the reaction and were analyzed with a Chemito GC1000 gas chromatograph equipped with a SE-30 capillary column (oven temperature $353-503 \mathrm{~K}$, injector temperature 373 $\mathrm{K}$ and detector temperature $373 \mathrm{~K}$ ). The products were further analyzed by GC-MS using a Shimadzu-5050 instrument provided with a $30 \mathrm{~m}$ HP-30 capillary column of cross linked 5\% phenyl methyl silicone.

\section{Results and discussions}

Wide angle XRD analysis clearly shows peaks corresponding to the crystalline cubic fluorite ceria phase (PDF-ICDD34-0394). With loading ofmetals no additional metal oxide phase was formed up to a $10 \mathrm{wt} \%$ metal loading except for copper. A peak at low-angle region confirms the mesoporous nature of the sample prepared (Fig. 1).

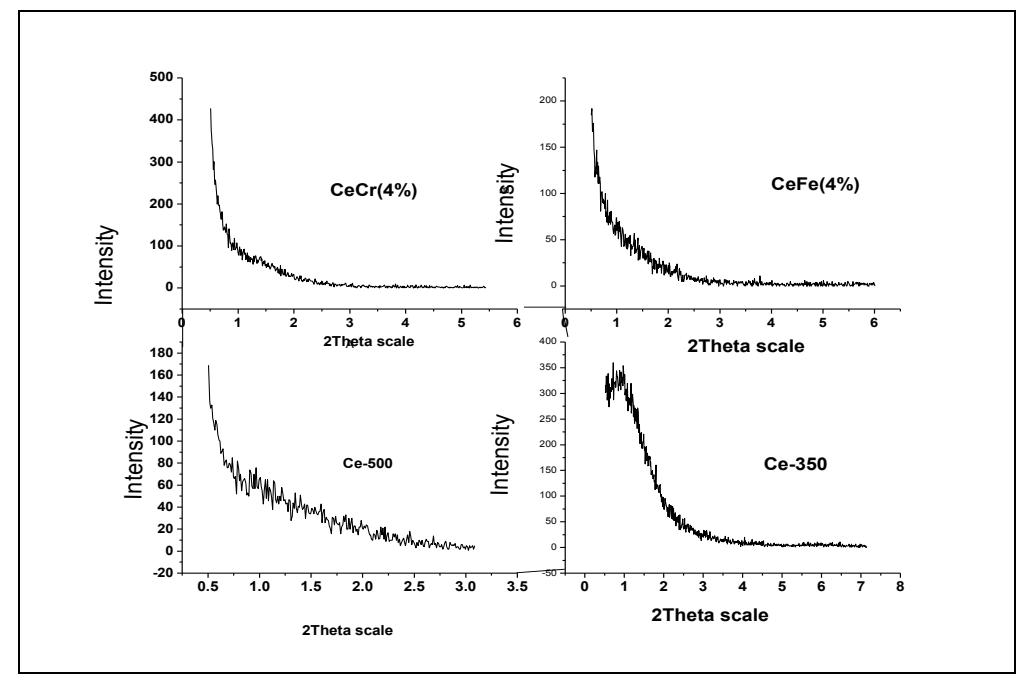

Fig. 1 Low-angle XRD pattern of mesoporous ceria and modified samples

The appearance of low-angle diffraction peaks indicates that mesoscopic order is preserved in the calcined metal oxide materials. The sample exhibits $\mathrm{N}_{2}$ adsorption-desorption of Type IV isotherm with steps between 

partial pressures $\mathrm{P} / \mathrm{Po}$ of 0.3 to 0.8 , and a hysteresis loop, due to capillary condensation in the mesoporous channels and/or cages (Fig.2).

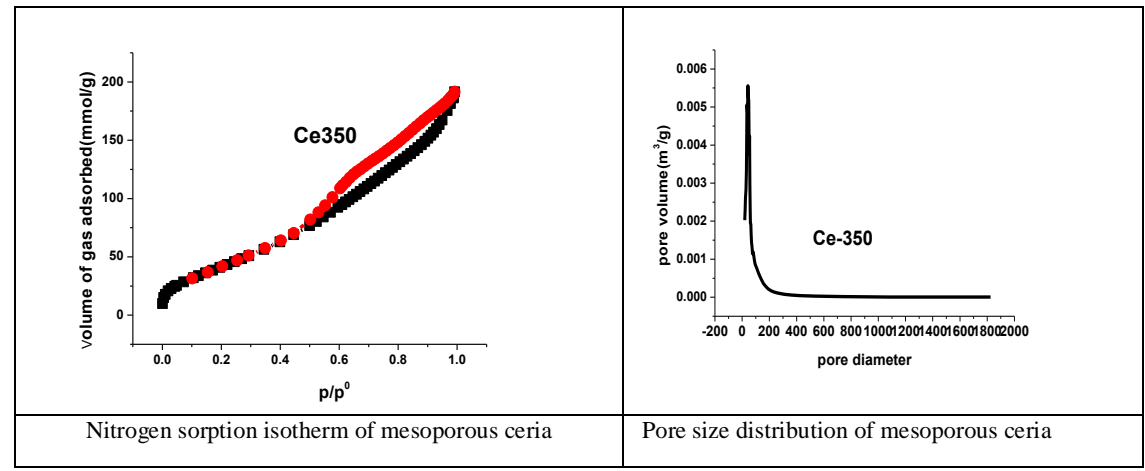

Fig. 2 Nitrogen sorption isotherms \& pore size distribution curves

The pore volume, pore diameter \& surface area calculated by desorption studies are given in (TABLE1).

Table.1 Surface area, Pore measurements

\begin{tabular}{|l|l|l|l|}
\hline sample & BET S.A $\left(\mathrm{m}^{2} \mathrm{~g}^{-1}\right)$ & $\begin{array}{l}\text { Average Pore } \\
\text { Diameter }(\mathrm{nm})\end{array}$ & $\begin{array}{l}\text { CumulativePore Volume } \\
\left(\mathrm{cm}^{3} \mathrm{~g}^{-1}\right)\end{array}$ \\
\hline $\mathrm{Ce} 350$ & 164 & 4.19 & 0.29 \\
\hline $\mathrm{CeFe}(2 \%)$ & 100 & 4.5 & 0.18 \\
\hline $\mathrm{CeFe}(4 \%)$ & 99 & 5.2 & 0.17 \\
\hline $\mathrm{CeFe}(10 \%)$ & 52.6 & 4.6 & 0.12 \\
\hline $\mathrm{CeCr}(2 \%)$ & 123 & 5.4 & 0.2 \\
\hline $\mathrm{CeCr}(4 \%)$ & 120 & 5.6 & 0.18 \\
\hline $\mathrm{CeCr}(10 \%)$ & 96 & 3.8 & 0.14 \\
\hline $\mathrm{CeNi}(2 \%)$ & 96 & 4.6 & 0.18 \\
\hline $\mathrm{CeNi}(4 \%)$ & 89 & 4.4 & 0.15 \\
\hline $\mathrm{CeNi}(10 \%)$ & 85 & 3.8 & 0.14 \\
\hline $\mathrm{CeCu}(2 \%)$ & 82 & 4.4 & 0.18 \\
\hline $\mathrm{CeCu}(4 \%)$ & 80 & 5.2 & 0.17 \\
\hline $\mathrm{CeCu}(10 \%)$ & 72 & 4.3 & 0.15 \\
\hline
\end{tabular}

From TABLE. 1 it is evident that surface area decreases with metal loading. Crystallite size and lattice parameters are calculated from XRD data (TABLE2).

Table 2. XRD data

\begin{tabular}{|l|l|l|l|}
\hline Sample & $\begin{array}{l}\text { Average } \\
\text { crystallitesize }(\mathrm{nm})\end{array}$ & Lattice parameter(nm) & d-spacing(nm) \\
\hline $\mathrm{Ce} 350$ & 12.8 & 0.545 & 3.15 \\
\hline $\mathrm{CeFe}(4 \%)$ & 10.26 & 5.39 & 3.12 \\
\hline $\mathrm{CeFe}(10 \%)$ & 10.66 & 5.44 & 3.15 \\
\hline $\mathrm{CeCu}(2 \%)$ & 6.93 & 5.41 & 3.13 \\
\hline $\mathrm{CeCu}(4 \%)$ & 11.5 & 5.41 & 3.13 \\
\hline
\end{tabular}




\begin{tabular}{|l|l|l|l|}
\hline & & & \\
\hline $\mathrm{CeCu}(10 \%)$ & 6.93 & 5.38 & 3.11 \\
\hline $\mathrm{CeNi}(2 \%)$ & 7.1 & 5.45 & 3.12 \\
\hline $\mathrm{CeNi}(4 \%)$ & 6.93 & 5.44 & 3.14 \\
\hline $\mathrm{CeNi}(10 \%)$ & 6.3 & 5.44 & 3.15 \\
\hline
\end{tabular}

In FT-IR spectrum for the calcined materials, bands related to the template are removed which shows the successful removal of surfactant at a lower calcination temperature $\left(350^{\circ} \mathrm{C}\right)$ which is effective for a high surface area for the calcined sample free of surfactant. No peaks corresponding to the $\mathrm{C}-\mathrm{H}$ bands are seen in calcined sample.

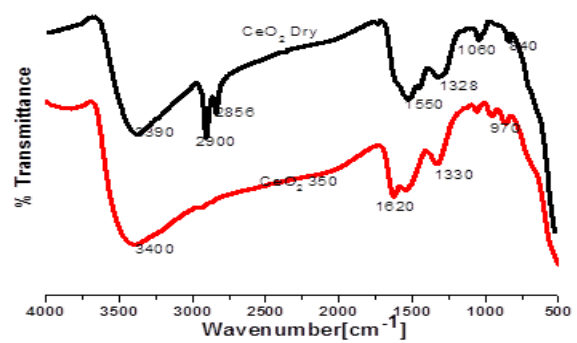

Fig.3 FT-IR spectrum of dried and calcined sample

HR-TEM(Fig.4) shows the formation of uniform crystallites of few nanometres in size

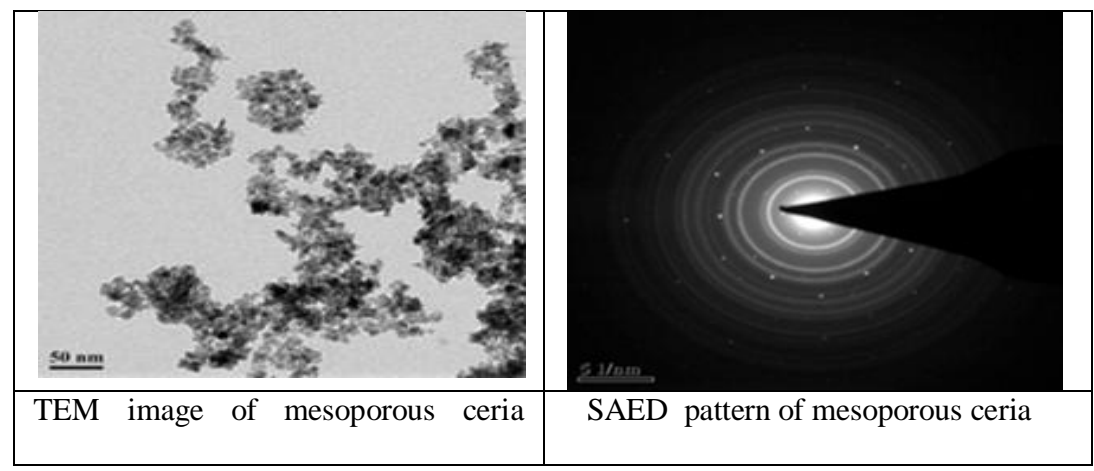

Fig. 4.TEM images of mesoporous ceria

The TG curves of pure ceria show weight loss near $100^{\circ} \mathrm{C}$ which is due to the loss of physisorbed water as well as water held on surface of the catalyst. The second weight loss around $125-315^{\circ} \mathrm{C}$ could be due to the elimination of surfactant which is occluded in the mesopores through oxidation process. No phase transition is observed indicating the stability of cerium oxide fluorite phase (Fig. 5)

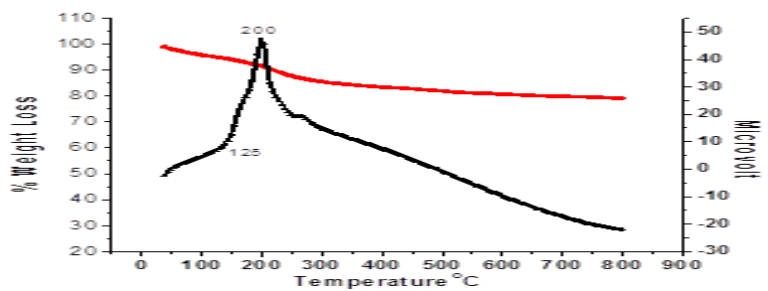

Fig. 5 TG/DTA spectrum of s ceria 
Table 3. Acidity values obtained from TPD of ammonia, \& vapour phase cumene cracking reaction

a The acidity values equivalent to desorbed ammonia, b The ratio of Lewis to Bronsted acidity obtained from the cumene cracking test reaction for acidity; c-In vapour phase cumene cracking test reaction

UV-Vis. DRS spectrum clearly shows that a single band arising from ceria species around $270 \mathrm{~nm}$ is found which shows that the $\mathrm{Ce}^{4+}$ species are in the tetra co-coordinated environment. The acidities of the samples measured by TPD of ammonia indicate an increase in the total acidity with the metal loading in the sample (TABLE3).

Vapour phase cumene cracking reaction is a model reaction for identifying the Lewis to Brönsted acid ratio of a catalyst. The major reactions taking place during the cracking of cumene are dealkylation to give benzene and propene over Bronsted acid sites (BAS) and dehydrogenation to give $\alpha$-methyl styrene over Lewis acid sites (LAS). A comparison of the amount of dealkylated products and $\alpha$-methyl styrene gives an idea about the (BAS) and (LAS) possessed by the catalyst [4].

The reaction conditions play an important role in deciding the catalytic activity. The factors influencing the activity of catalyst in the reaction were studied by varying the reaction temperature, weight of the catalyst, molar ratio of cyclohexanone to methanol and time of reaction. The parameters were optimized and the best conditions were found(TABLE 4).

Table4. Optimized Reaction conditions for acetalization of cyclohexanone

\begin{tabular}{|l|l|}
\hline Parameters & Optimized conditions \\
\hline Temperature & $70^{\circ} \mathrm{C}$ \\
\hline Cyclohexanone-Methanol Ratio & $1: 10$ \\
\hline Catalyst Amount & $0.05 \mathrm{~g}$ \\
\hline Time & $30 \mathrm{~min}$ \\
\hline
\end{tabular}

To test the heterogeneity of the catalytic system, the leaching test was performed. For this the catalyst was removed from the reaction mixture by filtration after an interval. Then the reaction was allowed to proceed again without the catalyst and the $\%$ conversion of cyclohexanone before and after the filtration was analysed using GC. The reaction was carried out at $70^{\circ} \mathrm{C}$, using $\mathrm{CeCr}(10 \%)$ keeping themolar ratio of cyclohexanone to methanol 1:10 for 15 minutes. Then the catalyst is filtered off and again the reaction mixture was allowed to proceed under the same condition for 15 more minutes. It was found that after filtration of the catalyst, the filtration liquor reacted much more slowly and at a similar rate to the reaction taking place in the absence of the catalyst (Table5, Entry2), indicating that no leaching of the active catalytic species occurred during the reaction.After filtration, the catalyst was washed with methanol and dried before the next cycle. The catalyst was found to be reusable for consecutive cycles without any significant loss of activity.

Table 5.Influence of metal leaching in acetalization of cyclohexanone with methanol

\begin{tabular}{|l|l|}
\hline Time (min) & Conversion $(\%)$ \\
\hline 15 & 48.4 \\
\hline $30 *$ & 49 \\
\hline
\end{tabular}

Catalyst: $0.05 \mathrm{~g}$ CeCr(10\%), Temperature: $70^{\circ} \mathrm{C}$, Cyclohexanone to methanol molar ratio: $1: 10$

\subsection{Catalytic Activity of prepared samples}

The conversion was analysed. The results are given in the TABLE.6

Table 6. Catalytic activities of different prepared catalysts

\begin{tabular}{|l|l|l|l|}
\hline Catalyst & $\begin{array}{l}\text { Conversion of } \\
\text { cy. hexanone }(\%)\end{array}$ & TON $^{\mathrm{a}}$ & TOF $^{\mathrm{b}}$ \\
\hline $\mathrm{CeO}_{2}$ & 12 & - & - \\
\hline w.c & 4 & - & - \\
\hline $\mathrm{CeFe}(2 \%)$ & 56.4 & 318.5 & 637 \\
\hline $\mathrm{CeFe}(4 \%)$ & 57 & 160.9 & 321.8 \\
\hline $\mathrm{CeFe}(10 \%)$ & 64 & 72.3 & 144.6 \\
\hline $\mathrm{CeCr}(2 \%)$ & 28 & 141.5 & 283 \\
\hline $\mathrm{CeCr}(4 \%)$ & 33.7 & 85.1 & 170.2 \\
\hline $\mathrm{CeCr}(10 \%)$ & 53.4 & 53.9 & 107.8 \\
\hline $\mathrm{CeNi}(2 \%)$ & 33.3 & 188 & 376 \\
\hline $\mathrm{CeNi}(4 \%)$ & 32.5 & 91.8 & 183.6 \\
\hline & & & \\
\hline
\end{tabular}


Acetalization Of Cyclohexanone Over Transition Metals Modified Mesoporous Ceria-A Green

\begin{tabular}{|l|l|l|l|}
\hline $\mathrm{CeNi}(10 \%)$ & 33.2 & 37.5 & 75 \\
\hline $\mathrm{CeCu}(2 \%)$ & 36 & 230.4 & 460.8 \\
\hline $\mathrm{CeCu}(4 \%)$ & 38.1 & 121.9 & 243.8 \\
\hline $\mathrm{CeCu}(10 \%)$ & 35.7 & 45.7 & 91.4 \\
\hline
\end{tabular}

a- TON $=($ mmoles of reagent reacted $/$ mmoles of metal added $) \times 100$

b- TOF $=$ TON / time $(h)$

In all the cases the corresponding diacetal was obtained as a single product with high yield. In a typical run, $10 \mathrm{ml}$ of 1:10 mixture of ketone and methanol was stirred with $50 \mathrm{mg}$ of pre-activated catalyst, at $70^{\circ} \mathrm{C}$ for 30 minutes.

For a comparison of catalytic activity of mesoporous ceria modified by different transition metals as catalyst for acetalization of cyclohexanone, reaction was studied both in presence of catalysts and without catalyst. A comparative evaluation result obtained is given in the TABLE3.

It is noticeable that a negligible conversion was obtained for blank reaction. Pure Mesoporous ceria gave very low conversion under the specified reaction conditions. Modification with different transition metals increases the activity in acetalization reaction. All the modified ceria catalysts produced dimethyl acetal as the only product during the reaction. The highest activity was shown by $10 \%$ iron incorporated mesoporous ceria system. , it appears that the reaction proceeds with the formation of very bulky intermediates and a microporous material like zeolite may be less reactive in comparison to a mesoporous material. It is not only the acidic structure of the catalysts which determine the acetalization ability to a greater extent but also the diffusional properties of the catalytic systems are the deciding factor in the acetalization reaction of ketones. The activity of catalyst towards the acetalization reaction does not require strong acidic sites [5]. As pointed out by Corma and co-workers the pore diffusion limitation imposed by larger molecular sizes $(0.75 \mathrm{~nm}$ for cyclohexanone as determined by the energy minimization program) of the reactant played an important role during the acetalization reaction using solid acid catalysts $[6,1,7]$. In the case of cyclohexanone; the distance between oxygen and the most distant hydrogen is $0.51 \mathrm{~nm}$; the van der Waals radius of oxygen is 0.14 , and that of hydrogen is $0.1 \mathrm{~nm}$ leading to an effective end-to-end distance of $0.75 \mathrm{~nm}$. Its volume is estimated at 38.351 $\mathrm{nm} 3$ [8]. Enhancement in catalytic activity could be explained in terms of improvement in textural and structural properties. Enhancement in the total pore volume could provide a better diffusional pathway for the bulky acetals. It appears that the acid structural properties and the diffusional properties of the catalytic systems are the deciding factor in the acetalization reaction of ketones.

The enhancement in catalytic activity could be explained in terms of improvement in textural and structural properties. Enhancement in the total pore volume could provide a better diffusional pathway for the bulky acetals. The enhanced formation of acetal over transition metal modified mesoporous ceria could also be explained as follows. In general transition metal modified mesoporous ceria possess much more amounts of weak plus medium acid sites and an assembly of these weak acid sites may act as an effective/strong acid site. A reasonable hypothesis is that all the surface hydroxyl groups on the pore wall of the catalyst point to the centre of each pore, and thus they could work as a group. Such an assembly might induce efficient catalysis despite the low acidity of each -OH group [9]. In the case of modified samples, the surface hydroxyl groups provide most of the weak acid sites.

The method developed by us does not involve additives and the catalysts can be recycled without loss of activity. Removal of water is not warranted in these reactions.

For the comparison of catalyst composition, from the product yield and considering the reaction as pseudo unimolecular, the intrinsic rate constants in the units of $\min ^{-1} \mathrm{~m}^{-2}$ was determined for each catalyst sample using the formula,

$\mathrm{k}\left(\min ^{-1} \mathrm{~m}^{-2}\right)=\frac{2.303}{\mathrm{tw} A} \log \frac{100}{100-\% \text { yield }}$

Where

$\mathrm{t}$ : Time of the reaction in minutes,

w: Weight of the catalyst (g), and

A: BET surface area of the catalyst $(\mathrm{m} 2 / \mathrm{g})$.

The rate constants of different samples are given in the TABLE 7 
Acetalization Of Cyclohexanone Over Transition Metals Modified Mesoporous Ceria-A Green

Table7. Rate constants of acetalization of cyclohexanone using different catalyst systems

\begin{tabular}{|l|l|}
\hline Catalyst & Rate constant $\times 10^{-3}\left(\mathrm{~min}^{-1} \mathrm{~m}^{-2}\right)$ \\
\hline $\mathrm{Ce}$ & 0.05 \\
\hline $\mathrm{CeFe}(2 \%)$ & 2.96 \\
\hline $\mathrm{CeFe}(4 \%)$ & 3.32 \\
\hline $\mathrm{CeFe}(10 \%)$ & 7.5 \\
\hline $\mathrm{CeCr}(2 \%)$ & 1.77 \\
\hline $\mathrm{CeCr}(4 \%)$ & 2.29 \\
\hline $\mathrm{CeCr}(10 \%)$ & 4.37 \\
\hline $\mathrm{CeNi}(2 \%)$ & 2.62 \\
\hline $\mathrm{CeNi}(4 \%)$ & 2.89 \\
\hline $\mathrm{CeNi}(10 \%)$ & 3.15 \\
\hline $\mathrm{CeCu}(2 \%)$ & 3.62 \\
\hline $\mathrm{CeCu}(4 \%)$ & 3.99 \\
\hline $\mathrm{CeCu}(10 \%)$ & 4.08 \\
\hline
\end{tabular}

The data reveal that the effect of modification of ceria with transition metals enhances its catalytic activity to a large extent. But the effect on catalytic activity by different metals is not alike. Different metals show different trend in the enhancement of catalytic activity.Acetalization reaction being an acid catalysed reaction, the difference in the activities of the catalysts can be attributed to their difference in the acidity values. TABLE.7. shows that the conversion of cyclohexanone upon acetalization, using different systems are in agreement with sum of weak and medium acidity as measured by ammonia TPD studies. The strong influence of textural properties of the catalysts such as acid amount and adsorption properties (surface area and pore volume) determine the catalytic activity. The reaction requires active sites with considerable acid strength. In addition to the acid strength, the pore volume, pore sizes and their distribution in the catalyst are responsible for their activity in the reaction.

\section{De-protection reaction (Hydrolysis of dimethoxy cyclohexanone)}

A major challenge in a multistep synthesis is to protect a carbonyl group from nucleophilic attack until its electrophilic properties of carbonyl can be exploited. Thus, regeneration of the parent carbonyl compound is a key step in such reactions. De-protection is often performed by acid-catalyzedtrans acetalization in acetone (in excess or as solvent), or hydrolysis in wet solvents or in aqueous acid. A convenient de-protection of acyclic and cyclic $\mathrm{o}, \mathrm{o}$-acetals and $\mathrm{o}$, o-ketals is achieved in excellent yields within minutes under neutral conditions in the presence of a catalytic amount of iodine [10]. Now we wish to report a convenient method for deprotection of acetals or ketals. Hydrolysis of acetal was performed at $70^{\circ} \mathrm{C}$ in the presence acetone-water mixture in the ratio 20:1. The reaction was conducted for 30 minutes by adding $10 \mathrm{ml}$ of the acetone - water mixture.

Table.8. Catalytic Activity of prepared samples in deacetalization of acetals

\begin{tabular}{|l|l|}
\hline Catalyst & \%Deacetalization \\
\hline $\mathrm{CeFe}(2 \%)$ & 100 \\
\hline $\mathrm{CeFe}(4 \%)$ & 100 \\
\hline $\mathrm{CeFe}(10 \%)$ & 100 \\
\hline $\mathrm{CeCr}(2 \%)$ & 100 \\
\hline $\mathrm{CeCr}(4 \%)$ & 98 \\
\hline $\mathrm{CeCr}(10 \%)$ & 98.2 \\
\hline $\mathrm{CeNi}(2 \%)$ & 100 \\
\hline $\mathrm{CeNi}(4 \%)$ & 100 \\
\hline $\mathrm{CeNi}(10 \%)$ & 100 \\
\hline $\mathrm{CeCu}(2 \%)$ & 100 \\
\hline $\mathrm{CeCu}(4 \%)$ & 100 \\
\hline $\mathrm{CeCu}(10 \%)$ & 100 \\
\hline
\end{tabular}




\section{Conclusion}

Neutral surfactant route using $\operatorname{HAD}$ (Hexa Decyl Amine) as surfactant is effective for the preparation of mesoporous ceria with high surface area and pore volume at a lower calcination temperature. Mesoporous ceria modified with $\mathrm{Fe}$ can be successfully utilized for protecting groups of carbonyl compounds during organic synthesis within $1 / 2$ hour using only $0.05 \mathrm{~g}$ of the catalyst under milder condition. The conversion is found to be correlated to the weak and medium acidity of the samples. Pure ceria gave low conversions than metal modified samples. The metal modification improves the $\%$ conversion to a great extent. The increase in $\%$ conversion and activity may be attributed to the increase in the acid sites upon metal modification. We introduced a mild and selective reagent for the oxidative cleavage of acetals under aqueous and heterogeneous conditions. The stability, simple work-up and high yields of the products are among the advantages of this procedure which make it an attractive supplement to the present reported methods.

\section{Acknowledgement:}

The financial support from University Grants Commission (India) to Rose Philo K.J to undergo Faculty Improvement Programme of UGC XI plan is gratefully acknowledged.

\section{References}

[1] T. W. Green, P. G. M. Wuts, "Protective Groups on Organic Synthesis", vol. 4, 2nd ed., Wiley, New York, (1991), 212

[2] P. T. Tanev, T. J. Pinnavaia, Science, 267, (1995), 865-867

[3] M. A. Zolfigol, Synth. Commun.30, (2000), 1593

[4] W. S. Johnson, C. A. Harbert, R. D. Stipanovic, J. Am. Chem. Soc.90, (1968), 5279

[5] M. J. Climent, A. Corma, S. Iborra, M.C. Navarro, PrimoJ, J. Catal. 161(1996)783.

[6] A. Corma, H. Garcia, Cat. Today 38, (1997), 257.

[7] R. Ballini, G. Bosica, B. Frullant, R. Maggi, G. Sartori, F. Schroer, Tetrahedron Lett. 39, (1998) 1615.

[8] B. Thomas et al. Microporous and Mesoporous Materials 80, (2005) 65-72

[9] A. Corma, M. J. Climent, H. Garcia, J. Primo, Appl. Catal. A: Gen. 59, (1990) 333

[10] J. Sun, Y. Dong, I.Cao, X. Wang, S. Wang, Y. Hu, J. Org. Chem., 69, (2004), 8932-8934. 